\title{
Medición del clima organizacional con un enfoque de género en la Escuela Politécnica Nacional
}

\author{
María Genoveva Espinoza-Santeli* \\ Andrea Angélica Jiménez Vera**
}

Fecha de recibido: 3 de mayo 2018 Fecha de aprobado: 16 de julio de 2018

Para citar: Espinoza Santeli, G. M., \& Jiménez Vera, A. A. (2019). Medición del clima organizacional con un enfoque de género en la Escuela Politécnica Nacional. Universidad \& Empresa, 21(36), 261-284. DOI: http://dx.doi.org/10.12804/revistas.urosario.edu.co/empresa/a.6778

* Licenciada en Ciencias Jurídicas, y Abogada, Pontifica Universidad Católica del Ecuador. Diploma Superior en Diseño, Gestión y Evaluación de Proyectos de Desarrollo, FLACSO, Quito, Ecuador. Magíster en Dirección de Negocios, con mención en Dirección Estratégica, Universidad Internacional del Ecuador, Quito. Ph.D en Economía de Población, Recursos y Ambiente, Zhongnan University of Economics and Law, Wuhan, China. Docente investigadora del Área de Gestión, Universidad Andina Simón Bolívar, sede Ecuador. Correo electrónico: genoveva.espinoza@uasb.edu.ec

** Ingeniera Empresarial, Escuela Politécnica Nacional, Quito, Ecuador. Docente universitaria y colaboradora en proyectos de investigación, Escuela Politécnica Nacional, Quito, Ecuador. Correo electrónico: andrea.jimenez01@epn.edu.ec 


\section{Resumen}

La investigación mide el clima organizacional percibido por los docentes de la EPN y determina si existen diferencias cuando se desagregan los resultados por género. La metodología es de naturaleza cuantitativa con una muestra de 61 docentes. La herramienta se construyó considerando dos factores: las dimensiones que son evaluadas con mayor frecuencia por instrumentos psicométricos validados de medición del clima organizacional y los parámetros que determinan discriminación laboral por género establecidos en el Modelo de Equidad de Género. Algunos resultados son: proporcionalmente existe una baja participación de mujeres que desempeñan labores de docencia y un menor porcentaje de contratos con nombramiento; existen diferencias marcadas en cuanto a sueldos y acceso a posiciones de poder; ninguna mujer gana más que la media de hombres y no hay mujeres en puestos directivos; en general, las mujeres consideran que el clima laboral no es adecuado en ninguna de las dimensiones consideradas -toma de decisiones, claridad organizacional, liderazgo y motivación-. En conclusión, la percepción del clima laboral difiere cuando se analizan los resultados desagregados por sexo; las mujeres perciben un clima organizacional inadecuado en tres de las dimensiones analizadas, además, se evidencia la existencia del techo de cristal en esta institución.

Palabras clave: género, clima organizacional, docentes y universidad.

\section{Measurement of Organizational Climate with a Gender Focus in the Escuela Politécnica Nacional Abstract}

This research measures the organizational climate perceived by teachers working at EPN and determines if there are differences when the results are disaggregated by gender. The methodology is of a quantitative nature with a sample of 61 teachers; the tool was constructed considering two factors: the dimensions that are most frequently evaluated by validated psychometric instruments for measuring the organizational climate and the parameters that determine labor discrimination by gender established in the Gender Equity Model. Some results are: there is a proportionally low participation of women who perform teaching tasks and a lower percentage of contracts with appointment; there are marked differences in salaries and in having access to positions of power; no woman earns more than the average of men and there are no women in management positions. In general, women consider that the work environment is not adequate in any of the dimensions considered decision making, organizational clarity, leadership and motivation. In conclusion, the perception of the work environment differs when the results disaggregated by sex are analyzed and the existence of the glass ceiling in this institution is evidenced.

Keywords: Gender, organizational climate, teachers, university.

\section{Medição do clima organizacional com um enfoque de gênero na Escola Politécnica Nacional}

\section{Resumo}

A pesquisa mede o clima organizacional percebido pelos docentes da EPN e determina se existem diferenças quando se desagregam os resultados por gênero. A metodologia é de natureza quantitativa com uma amostra de 61 docentes; a ferramenta se construiu considerando dois fatores: as dimensões que são avaliadas com maior frequência por instrumentos psicométricos validados de medição do clima organizacional e os parâmetros que determinam discriminação laboral por gênero estabelecidos no Modelo de Equidade de Gênero. Alguns resultados são: proporcionalmente existe uma baixa participação de mulheres que desempenham labores de docência e uma menor percentagem de contratos com nomeação; existem diferenças marcadas em quanto a salários e acesso a posições de poder; nenhuma mulher ganha mais que a média de homens e não há mulheres em posições diretivas, em geral, as mulheres consideram que o clima laboral não é adequado em nenhuma das dimensões consideradas -tomada de decisões, claridade organizacional, liderança e motivação-. Em conclusão, a percepção do clima laboral difere quando se analisam os resultados desagregados por sexo, as mulheres percebem um clima organizacional inadequado em três das dimensões analisadas; além disso, se evidencia a existência do teto de cristal nesta instituição.

Palavras-chave: gênero, clima organizacional, docentes, universidade. 


\section{Introducción}

La gestión del clima organizacional (CO) es un compromiso y una corresponsabilidad para mejorar la calidad de vida de las personas que pertenecen a una organización. Un buen clima organizacional genera compromiso, motivación, desempeño, satisfacción y eficiencia en los colaboradores (Vega, Partido \& Rivera, 2015; Caligiore Corrales \& Díaz Sosa 2003; García Solarte, 2011; Chiang Vega, Núñez Partido \& Huerta River, 2012) y permite generar espacios laborales apropiados que inciden en la productividad de la organización (Vega, Rodrigo \& Partido, 2010; Salazar Estrada, Guerrero Pupo, Machado Rodríguez \& Cañedo Andalia, 2009; Torrecilla, 2005). Según Arciniegas (2002) cuando un docente universitario tiene un clima organizacional satisfactorio, tanto su productividad como el grado de compromiso aumentan, y se registran bajas tasas de absentismo.

Ante las nuevas exigencias sociales, las instituciones de educación superior cumplen con un papel fundamental para la sociedad, pues responden a las demandas actuales de formación profesional y técnica, además de contribuir con la formación de ciudadanos conscientes, críticos, multidisciplinarios y éticos. En este contexto, los docentes son un pilar fundamental para alcanzar y satisfacer dichas expectativas, por lo que el CO en el que se desempeñan debe brindar un espacio de trabajo favorable, sano, estimulante y atractivo para poder llevar a cabo las actividades de gestión académica, docencia, investigación y vinculación con la colectividad, es decir, las universidades deben intentar ser organizaciones óptimas que provean un clima laboral que permita satisfacer las necesidades de sus empleados -docentes y administrativos- y alumnos.

La presente investigación realiza una medición del clima organizacional de uno de los más antiguos e importantes centros de educación superior de ciencias duras del Ecuador, la Escuela Politécnica Nacional (EPN), tradicionalmente dirigida por hombres. La pregunta guía de investigación fue: ¿existe una diferencia significativa sobre la percepción del clima organizacional si se analiza la información desagregada por género en cada uno de las dimensiones? El documento está compuesto por cuatro acápites: presentación de la literatura relevante en torno a clima organizacional, enfoque de género y discriminación laboral por género, metodología -naturaleza, alcance, diseño, herramientas y método de análisis-; presentación y discusión de resultados y conclusiones. 


\section{Revisión de literatura}

El marco teórico que se presenta a continuación permite conocer y entender los conceptos, ideas y teorías relacionadas directamente con el objeto de estudio que es la medición del clima organizacional en las cuatro dimensiones seleccionadas: a) toma de decisiones, b) claridad organizacional, c) liderazgo y d) motivación. A continuación se presenta una síntesis de las herramientas psicométricas de medición del CO, se definen las prácticas discriminatorias por género, ${ }^{1}$ y se explica el enfoque de género mediante las dimensiones del Modelo de Equidad de Género (MEG) que mide la discriminación laboral por género.

\subsection{Clima organizacional}

Las definiciones sobre CO están agrupadas bajo tres enfoques: estructuralista, subjetivo y de síntesis; Pérez de Maldonado, Maldonado Pérez y Bustamante Uzcátegui (2006) realizaron una sistematización de varios conceptos según los tres enfoques, como se puede apreciar en la tabla 1.

Tabla 1. Enfoques del clima organizacional

\begin{tabular}{|c|c|c|}
\hline Enfoque & $\begin{array}{c}\text { Principales } \\
\text { autores }\end{array}$ & Definición de clima organizacional \\
\hline 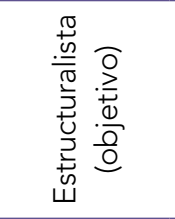 & $\begin{array}{c}\text { Forehand y } \\
\text { Gilmer }\end{array}$ & $\begin{array}{l}\text { Conjunto de características permanentes que describen una organización, la distinguen } \\
\text { de otra e influyen en el comportamiento de las personas que la forman. La perspectiva del } \\
\text { individuo depende del ambiente en el cual se encuentre; hace énfasis en las características } \\
\text { permanentes de una organización que la diferencian de otra e influyen en el comportamiento } \\
\text { de las personas. }\end{array}$ \\
\hline \multirow[b]{2}{*}{ 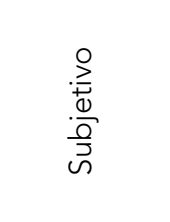 } & \multirow[b]{2}{*}{ Halpin y Crofts } & La opinión que el trabajador se forma de la organización. \\
\hline & & $\begin{array}{l}\text { Concibe la problemática a partir de la percepción y opinión que el trabajador tiene de } \\
\text { la organización. Se considera que el comportamiento de los trabajadores no es una } \\
\text { consecuencia de los factores organizacionales existentes, sino de las percepciones que el } \\
\text { trabajador tiene de estos factores, que a su vez está influida por su carga personal cultural. }\end{array}$ \\
\hline \multirow[b]{2}{*}{ 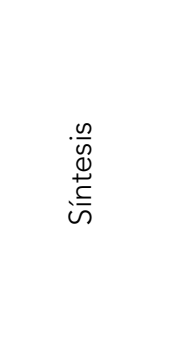 } & & $\begin{array}{l}\text { Efecto subjetivo percibido del sistema, que forman el estilo informal de los administra- } \\
\text { dores y de otros factores ambientales importantes sobre las actividades, las creencias, } \\
\text { los valores y la motivación de las personas que trabajan en una organización dada. }\end{array}$ \\
\hline & $\begin{array}{l}\text { Litwin y } \\
\text { Stringer }\end{array}$ & $\begin{array}{l}\text { En este enfoque se plantean los efectos subjetivos percibidos sobre el estilo administrativo de } \\
\text { una organización, pero también otros factores ambientales sobre las actividades, creencias, } \\
\text { valores y motivación de las personas que trabajan en una determinada organización; es decir, } \\
\text { la percepción que una persona tiene de una organización depende de las interacciones y } \\
\text { convivencia que experimenta }\end{array}$ \\
\hline
\end{tabular}

Fuente: elaboración propia con base en Pérez de Maldonado, Maldonado Pérez y Bustamante Uzcátegui (2006).

1 Se considera relevante la diferenciación entre sexo y género, mientras que la primera hace referencia a las características físicas, naturales y biológicas, el segundo es una construcción social que se produce culturalmente en base al sexo y que permite asignar diferentes roles y valores según cada cultura. 
El elemento perceptual es el constructo que se utiliza con mayor frecuencia por varios estudiosos del clima organizacional, ya que al hablar de percepciones se hace posible una medición mediante encuestas, que es la herramienta adoptada en esta investigación. "La percepción que los sujetos hacen de la situación está influenciada por cuestiones internas y externas a ellos. Las internas están relacionadas con las dimensiones personales, como los aspectos psíquicos y anímicos e institucionales de la organización, y los externos como familiares, sociales, de educación y económicos que rodean la vida de cada individuo, condicionando su visión del clima" (Cano-García, Padilla-Muñoz \& Carrasco-Ortiz, 2005, citados por Rodríguez Mantilla \& Fernández Díaz 2015, p. 73).

Así, para la presente investigación se consideró el enfoque subjetivo para la definición de clima organizacional, es decir, el planteado por Halpin y Crofts (1962). Complementariamente, se considera la definición de Guerra, Vargas, Castro, Plaza y Barrera (2012), quienes definen al clima organizacional como la percepción que tienen los sujetos sobre las relaciones interpersonales que se establecen en el lugar de trabajo y se constituye en el marco dentro del cual se desarrollan dichas interacciones, es decir, el $\mathrm{CO}$ es la forma en que cada uno percibe el mundo dentro de su contexto e influye en el comportamiento del individuo al interior de la organización y, en el caso de análisis, la percepción que tienen los docentes de la EPN.

El clima organizacional resulta ser un enfoque y una herramienta administrativa importante en la toma de decisiones de los directivos y en la dinámica organizacional, pues incide sobre el clima de la organización, ya que son más que percepciones que generan diferentes apreciaciones asociadas a valores, posiciones ideológicas, acceso al poder y a toma de decisiones, normas establecidas, institucionalidad de la organización y prácticas intrínsecas; este conjunto de variables se traduce en pautas de comportamiento que, a su vez, se diferencian si se considera el género y sexo de los involucrados.

Para Merleau-Ponty (1975) la percepción es un proceso parcial de creación de juicios, debido a que el observador no siempre percibe las cosas en su totalidad; este escenario se presenta porque las situaciones, perspectivas y cosmovisiones en las que se desarrollan las sensaciones dependen del contexto -entorno que además es percibido de forma diferente por hombres y mujeres- y lo que se obtiene es solo un aspecto de los objetos en un momento determinado, otorgando a la percepción tres características: subjetiva, selectiva 
y temporal. Por tanto, la apreciación que tienen los miembros sobre una organización (Gül 2008), de tener un impacto positivo, implicará un mayor compromiso y lealtad de los colaboradores hacia la organización.

El clima organizacional está estrechamente vinculado con investigaciones cuantitativas que analizan las percepciones, actitudes y comportamientos de los individuos, pero para poder medirlo hay que llegar a un consenso sobre cuáles son las dimensiones que se quieren medir, y para eso, primero hay que determinar cuál es el enfoque de CO que se quiere seguir; en un segundo punto, es necesario determinar si existen otros constructos teórico-sociales que son importantes. Para el caso, se considera que el enfoque pertinente es el subjetivo, porque lo más importante es la percepción que cada individuo tiene sobre su lugar de trabajo, eso con el fin de poder incorporar posteriormente el enfoque de género. Peiró (1995) afirma que para mejorar la organización es necesario ajustar y mejorar la interacción hombre-organización, pero para incluir variables que permanecen fuera del mainstream hay que incorporar el enfoque de género para la medición del clima organizacional. Puede que, en términos generales o en promedios, el CO de una organización sea aceptable, pero ¿qué pasa cuando esos mismos resultados son desagregados por género?

\subsection{Herramientas psicométricas}

Las herramientas de medición del clima laboral -herramientas psicométricas- permiten medir características psicológicas o, en este caso, medir la calidad del clima organizacional en varias dimensiones. La dificultad aparece cuando no existe un consenso sobre las dimensiones que deberían medirse. Debido a la falta de consenso sobre las dimensiones que componen el clima organizacional, se han desarrollado una infinidad de instrumentos, cada uno con dimensiones a ser analizadas que concuerdan con los constructos del enfoque de CO. Cardona Echeverri y Cruz Zambrano (2014) analizan la estructura, dimensiones y validez de instrumentos psicométricos de los últimos 10 años, que tienen altos niveles de confiabilidad y validez para medir el clima organizacional, verifican la fundamentación de los constructos teóricos y establecen características psicométricas de las pruebas de evaluación del clima organizacional. Los instrumentos, en conjunto, estudian 21 dimensiones: interacción social/relaciones, sistema de recompensas e incentivos, apertura organizacional, supervisión, relación individuo-institución, logística institucional/inducción, equidad institucional, autorreflexión, sensación de seguridad, conflictos, aspectos físicos, apertura/tolerancia, compartir información, centralización, 
comunicación, humor, rumores/hipocresía, control, valores y cultura, comportamiento individual, compromiso con la productividad, aplicación al trabajo, involucración en el cambio, compatibilización de intereses, innovación y relación aprendizaje-apoyo, toma de decisiones/autonomía/responsabilidad, claridad organizacional/estructura organizacional; liderazgo/relaciones con jefes, y motivación institucional.

Las dimensiones que tienen una mayor frecuencia en todas las herramientas son toma de decisiones/autonomía/responsabilidad (8), claridad organizacional/estructura organizacional (8), liderazgo/relaciones con jefes (7) y motivación institucional (5). Según los autores, se definen como:

- Toma de decisiones: "percepción que se tiene de la capacidad para decidir sobre cuál es la alternativa más viables según las consideración de las personas en las organizaciones”.

- Claridad organizacional: "percepción que se tiene acerca de las alineaciones administrativas y su adecuación en la organización”.

- Liderazgo: "percepción que existe entre los directivos y las personas, con énfasis en la consideración que se tiene del direccionamiento dado a la organización".

- Motivación: "percepción que se tiene respecto a las posibilidades de la institución de dar un mejor acompañamiento para la consecución de las metas laborales y personales" (Cardona Echeverri \& Cruz Zambrano 2014, pp. 186-187).

\subsection{Perspectiva de género}

Para Fainstain y Perrota (2011) la perspectiva de género -género entendido como una construcción social- permite interpretar la realidad social, de tal manera que visibiliza y desnaturaliza los condicionamientos socioculturales que establecen desigualdades de poder. Se realizan investigaciones con enfoque de género con la finalidad de promover sociedades más equitativas y eliminar situaciones provocadas por la heteronormatividad, que se traducen en la vida diaria en actitudes de opresión, injusticia, subordinación y discriminación hacia las mujeres.

Para incluir la perspectiva de género en la investigación, se utilizó como herramienta el Modelo de Equidad de Género (Instituto Nacional de Mujeres de México 2013), sistema de gestión que plantea la posibilidad de alcanzar la igualdad de oportunidades y de trato entre 
mujeres y hombres en las organizaciones mediante la implementación de compromisos, análisis de percepción, diagnóstico inicial y acciones precisas (Jiménez, 2017).

El diagnóstico del Modelo de Equidad de Género (MEG) contempla categorías como: respeto a opiniones y aportaciones; acceso a información necesaria para desempeñar su trabajo; comunicación oportuna sobre asuntos importantes de la organización y sobre las expectativas y responsabilidades que se tiene sobre el empleado; evaluaciones objetivas y equitativas de desempeño; apoyo y retroalimentación para mejorar el desempeño; trato justo, canales de quejas y reclamos seguros y confiables; ambiente laboral amistoso, cordial, comprensible y solidario; justicia e igualdad en la división de cargas de trabajo y en la asignación de responsabilidades; recompensas y reconocimiento por trabajo bien desempeñado; superiores accesibles; preocupación por el bienestar personal y emocional del empleado; ascensos meritorios; existencia y respeto a planes de carrera y desarrollo profesional; pago justo por cargo y responsabilidades; espacio libre de favoritismos; trato justo e igualitario; ambiente libre de acoso laboral y sexual; adecuada planeación y distribución del trabajo en cuanto a número de niveles jerárquicos a los que reporta sobre la rutina y monotonía del trabajo y exceso de responsabilidad o exceso de funciones que se le asignan; posibilidad de solicitar permisos para ausentarse dentro del horario laboral para atender situaciones particulares, familiares $o$ escolares de carácter extraordinario y si se les anima a que concilien su vida laboral y personal.

\subsection{Discriminación laboral por género}

La discriminación puede presentarse en cualquier espacio, sea este laboral, familiar, social o educativo, y está presente en la vida cotidiana como una situación perjudicial para determinados colectivos o individuos que "al establecer una diferenciación por diversas razones, tiene como resultado un trato no igualitario" (Kurczyn, 2004, p. 34). Toda discriminación presenta una característica común, "supone dispensar un trato distinto a las personas atendiendo a una serie de características como sexo, ascendencia étnico-racial, orientación sexual, edad, religión, ideología, discapacidad, entre otras, lo cual entraña un menoscabo de la igualdad de oportunidades y de trato” (Instituto Nacional de Mujeres, 2013, p.12).

La discriminación por género se refiere a la separación de roles que se da a cada persona a partir de su sexo biológico. La diferencia sexual se convierte en una desigualdad social que otorga, de manera supuestamente natural, funciones separadas para hombres y mujeres, se construyen relaciones de poder y se da preferencia a ciertos grupos; de esta manera, el 
uso del género explica las asimetrías de las relaciones sociales y de poder en los distintos ámbitos del quehacer social y humano (Consejo Nacional para la Igualdad de Género, 2014). No se puede dejar de lado las instituciones de educación superior, que representan un espacio de interacción y creación de relaciones sociales, culturales y de poder, donde se puede decir que aún se mantienen estereotipos tradicionales basados en el sexo.

La discriminación laboral se presenta cuando dos personas con la misma capacidad y competencia para desempeñar un trabajo son tratadas como miembros de grupos diferentes y con reconocimientos distintos, ya sea en la remuneración o en las condiciones de trabajo. En la discriminación laboral por género, según Kurczyn (2004), ocurre una distinción o alteración de las condiciones en el trabajo entre hombres y mujeres donde, generalmente, los hombres son quienes tienen mejores condiciones laborales, ya sea oportunidades para acceder a un puesto de trabajo, de trato, de ascensos, prestaciones o en la cantidad de dinero que percibe por su trabajo, y asevera que la discriminación puede aplicarse tanto para suprimir derechos como para otorgar privilegios. En base a una investigación (Jiménez, 2017) se identificó que las prácticas y actitudes más comunes que generan discriminación laboral por género en las universidades públicas del DMQ impiden lograr un contexto de igualdad en todos los puntos señalados, como segregación ocupacional, brecha salarial, acoso sexual y acoso psicológico.

Una de las instituciones donde se sigue manteniendo una fuerte estructura patriarcal y vertical son los centros de educación superior. Para Gonzáles (2002), la universidad ecuatoriana es una organización con una estructura tradicional, que ha evolucionado desde una exclusión total, cuando solo se aceptaba hombres en las universidades -para estudiar y para desempeñar papeles administrativos y académicos- hasta una exclusión velada y simbólica, donde las mujeres pueden participar del derecho universal a la educación y acceso a puestos de trabajo, pero generalmente alejadas de los centros de poder y del proceso de toma de decisiones (Jiménez, 2017).

El clima organizacional como una percepción, también está relacionado con el género y a diferentes apreciaciones con relación al acceso a oportunidades en cuanto a cargos de responsabilidad o poder, promociones, puestos y expectativas por parte de los miembros de las organizaciones y brechas salariales, entre otras, que forman una percepción, en muchos casos opuesta por parte de hombres y mujeres en un mismo entorno laboral. 
Las universidades, en palabras de Ibarra, son como "anarquías organizadas y como unidades que compiten en mercados asimétricos que escapan a su control" (2002, citado en Lopera Palacio, 2004); tienen un alto impacto en la sociedad y son el eje fundamental de la sociedad del conocimiento, conformado por un grupo de personas adscritas a la organización en la que interactúan desde diferentes posiciones, sea como personal administrativo, docentes y técnicos, cada uno con sus intereses, necesidades y percepciones del entorno laboral, que a su vez deben interactuar con los estudiantes que son tanto su insumo como su cliente.

Acker, en su texto Género y educación: Reflexiones sociológicas sobre mujeres enseñanza y feminismo (1995), analiza la discriminación de las mujeres en el mundo académico como docentes e investigadoras, y llega a la conclusión de que la devaluación a nivel profesional de la mujer en las instituciones de educación superior es paralela a su feminización; además, las desigualdades en oportunidades de optar por una plaza, ascensos, tipo de jornada, asignación a cargos de responsabilidad -con su respectiva carga de poder- o titularidad se reproduce en la academia; es decir, las mujeres en la academia todavía tienen restricciones visibles como el limitado acceso a puestos directivos e inclusive, se evidencian sesgos en la asignación de cupos para dictar materias; sigue primando la dicotomía público/privado y la asignación de roles para decidir que cátedras son asignadas a las docentes mujeres, sin considerar el entorno, su formación general y las expectativas que tienen con relación a su crecimiento profesional. En palabras de Gil Lacruz "el género sigue siendo un principio organizador importante aplicable a asignaturas, prácticas administrativas, espacios, uniformes, rituales, división del trabajo entre el profesorado" (2008, p. 112).

El enfoque de género implica orientar primero el análisis de las relaciones entre los géneros y luego tomar acciones si estás relaciones generan inequidades. La perspectiva de género es una forma de ver y entender el mundo, tomando en cuenta las desigualdades de poder existentes entre los géneros, rescatando y visibilizando sus necesidades diferenciadas. Por lo tanto, implica reconocer que una cosa es la diferencia sexual y otra son las atribuciones, ideas, representaciones y prescripciones sociales que se construyen tomando como referencia a esa diferencia sexual. Todas las sociedades estructuran su vida y construyen su cultura en torno a la diferencia sexual; esta diferencia anatómica se interpreta como una diferencia sustantiva, que marcará el destino de las personas. Lo 
lógico, se piensa, es que si las funciones biológicas son tan dispares, las demás características -morales, psíquicas- también lo habrán de ser.

\section{Metodología}

La naturaleza de la investigación es cuantitativa, con un alcance descriptivo correlacional, el diseño es no experimental transversal, la herramienta es una encuesta y el método de análisis es estadístico inferencial y correlacional, en el que se busca establecer relaciones entre las dimensiones del clima organizacional y la diferencia de percepciones según el género de los encuestados.

Para determinar el número de encuestas a realizar se utilizó el muestreo aleatorio simple, con afijación proporcional; la información se obtuvo del distributivo de personal del año 2016 publicado en la página web de la universidad, donde se identificó que de 1457 (N) personas que laboran en la EPN, 737 son docentes $\left(\mathrm{N}_{\text {Docentes }}\right)$. Para realizar los cálculos se utilizó la siguiente fórmula:

$$
\text { (1) } \quad n=\frac{z^{2} p q N}{E^{2} N+z^{2} p q}
$$

Dónde: $\mathrm{N}_{\text {Docentes }}$ son 737 , el nivel de confianza del $95 \%(\mathrm{z}=1,96)$ con un error de muestra (E) del $5 \%$; como no se conocía la proporción de individuos que responderían con un sí o un no a una determinada pregunta, se consideró que p y q tomen el valor de 0,5 cada una. El resultado son 61 docentes $(n=61)$.

Se recogieron los datos de los docentes de la EPN mediante el uso de un sistema informático de encuestas en línea para permitir la confidencialidad y facilitar la sistematización de la información con el programa estadístico SPSS.

La encuesta tienen una doble construcción: en primer lugar, para analizar el clima organizacional, se realizó una búsqueda bibliográfica, que tuvo como resultado el hallazgo del artículo de Cardona Echeverri y Cruz Zambrano (2014), las dimensiones seleccionadas son las que aparecen con mayor frecuencia en todos los instrumentos de medición: toma de decisiones, claridad organizacional, liderazgo y motivación. La segunda consideración 
para la construcción de las preguntas fue el componente de perspectiva de género, para el cual se utilizó el esquema del MEG. Con esta información levantada se plantearon las preguntas que conformaron la encuesta. En la tabla 2 se puede apreciar lo antes expuesto, así como el desarrollo de las once preguntas:

Tabla 2. Componentes y criterios de encuesta de la investigación

\begin{tabular}{|c|c|c|c|c|}
\hline & $\frac{: 0}{\frac{0}{n}}$ & $\begin{array}{l}\text { Concepto de la } \\
\text { dimensión }\end{array}$ & Parámetros del MEG & Pregunta \\
\hline 1 & 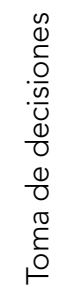 & $\begin{array}{l}\text { Considera la capacidad } \\
\text { para decidir sobre asuntos } \\
\text { de la organización y } \\
\text { cuanto influyen estas } \\
\text { decisiones en la misma. }\end{array}$ & $\begin{array}{l}\text { Lo considera de manera } \\
\text { cualitativa en la categoría } \\
\text { de discriminación en la } \\
\text { organización y toma en } \\
\text { cuenta otros aspectos } \\
\text { como las evaluaciones } \\
\text { del desempeño. }\end{array}$ & $\begin{array}{l}\text { 1. ¿Considera que se toman en cuenta sus opiniones } \\
\text { y comentarios? } \\
\text { 2. ¿Considera que se le comunica asuntos } \\
\text { importantes? Por ejemplo, asuntos que modifican } \\
\text { la vida laboral }\end{array}$ \\
\hline 2 & $\begin{array}{l}\overline{0} \\
\frac{0}{0} \\
\frac{0}{U} \\
\frac{\pi}{N} \\
\frac{N}{C} \\
\frac{0}{0} \\
\frac{0}{0} \\
\frac{0}{0} \\
\frac{\pi}{0} \\
\frac{0}{2} \\
\frac{0}{U}\end{array}$ & $\begin{array}{l}\text { Considera la información } \\
\text { con que se cuenta. } \\
\text { La relación que surge } \\
\text { entre las personas } \\
\text { con la organización } \\
\text { está orientada por la } \\
\text { intencionalidad de las } \\
\text { necesidades e intereses } \\
\text { de las partes. }\end{array}$ & $\begin{array}{l}\text { Lo considera de } \\
\text { manera cualitativa } \\
\text { en la categoría de } \\
\text { discriminación en la } \\
\text { organización y toma } \\
\text { en cuenta también } \\
\text { reconocimientos, pagos } \\
\text { y favoritismos. }\end{array}$ & $\begin{array}{l}\text { 3. ¿Considera que se le proporciona la información } \\
\text { necesaria para desempeñar sus labores, así como } \\
\text { una retroalimentación adecuada? } \\
\text { 4. ¿Considera que las responsabilidades de cada } \\
\text { puesto están claramente definidas? } \\
\text { 5. ¿Considera que existe igualdad en la división de } \\
\text { cargas de trabajo yasignación de responsabilidades? } \\
\text { 6. ¿Considera que los ascensos son otorgados a las } \\
\text { personas que más se lo merecen y están capacitadas? }\end{array}$ \\
\hline 3 & $\begin{array}{l}\text { O } \\
\text { N } \\
\frac{0}{0} \\
\frac{0}{0} \\
\frac{0}{1}\end{array}$ & $\begin{array}{l}\text { Función del líder, } \\
\text { relacionada con la } \\
\text { supervisión y requisición, } \\
\text { transmitida a partir de } \\
\text { directrices propias de la } \\
\text { organización. Habilidad } \\
\text { social que se traduce en } \\
\text { destrezas y competencias } \\
\text { profesionales que redundan } \\
\text { en el rendimiento, eficiencia } \\
\text { y eficacia. }\end{array}$ & $\begin{array}{l}\text { Lo considera de } \\
\text { manera cualitativa } \\
\text { en la categoría de } \\
\text { discriminación en la } \\
\text { organización. }\end{array}$ & $\begin{array}{l}\text { 7. ¿Los superiores o jefes le comunican sus expec- } \\
\text { tativas? } \\
\text { 8. ¿Considera que los superiores son accesibles y es } \\
\text { fácil hablar con ellos? }\end{array}$ \\
\hline 4 & 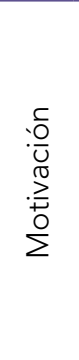 & $\begin{array}{l}\text { Considera el acompaña- } \\
\text { miento e incentivos que la } \\
\text { organización ofrece a sus } \\
\text { trabajadores. }\end{array}$ & $\begin{array}{l}\text { Lo considera de ma- } \\
\text { nera cualitativa en la } \\
\text { categoría de análisis } \\
\text { de la calidad de vida } \\
\text { organizacional. }\end{array}$ & $\begin{array}{l}\text { 9. ¿Considera que la organización se interesa por su } \\
\text { bienestar personal y emocional no solo como em- } \\
\text { pleado? } \\
\text { 10. ¿Se le otorgan reconocimientos por un trabajo } \\
\text { bien desempeñado? } \\
\text { 11. ¿Considera que debería ocupar un puesto superior } \\
\text { dentro de la organización por las responsabilida- } \\
\text { des que tiene? }\end{array}$ \\
\hline
\end{tabular}

Fuente: elaboración propia. 
La encuesta tuvo un proceso de validación de forma y fondo con especialistas en diseño de encuestas y gestión del talento humano de la EPN y de la Universidad Andina Simón Bolívar, sede Ecuador. La herramienta final es una encuesta conformada por 11 preguntas y los docentes respondieron según la Escala de Likert con cinco categorías de decisión: siempre (1), casi siempre (2), algunas veces (3), pocas veces (4) y nunca (5). Se consideró el promedio de tres como una respuesta neutral "ni de acuerdo ni en desacuerdo" (algunas veces, en este caso), que es la posición central de la escala, es asociada con un acuerdo moderado. Adicional al puntaje que cada individuo asignó, cada pregunta tenía un espacio para comentarios.

\section{Presentación y discusión de resultados}

En la primera parte se presentan los estadísticos descriptivos de la muestra con la siguiente información: edad, sexo, escolaridad, estado civil, hijos, permanencia en la institución, tipo de relación laboral, cargo y salarios; en la segunda parte, se presentan los resultados obtenidos a partir de la aplicación de la encuesta.

Los estadísticos descriptivos de la población encuestada son: de los 61 encuestados el $26,23 \%$ son mujeres y el 73,77 \% son hombres. La mayoría tiene entre 30 y 39 años, con el $36,07 \%$, seguido por el grupo que tiene entre 18 y 29 años con el 27,87\%. Al desagregar por sexo, las mujeres docentes se concentran en el grupo de 40 y 49 años, mientras que los docentes hombres se concentran en el grupo de 60 años en adelante, pues en el último grupo de edad no existen mujeres. Esta distribución de edades, donde las docentes jóvenes predominan en los primeros rangos de edad y se reducen en los últimos demuestra, por una parte, que se han integrado de manera más reciente y por otra que son los hombres los que continúan trabajando a avanzada edad, por lo que se comprueba que tienen mayor oportunidad de hacer una carrera a pesar de la edad.

En cuanto a la escolaridad, predominan los docentes que han obtenido una maestría con el 63,93 \%, mientras que quienes tienen una ingeniería o licenciatura alcanzan el 22,95\%. Uno de los factores para que la mayoría de los docentes tenga un título de maestría es que, según el Reglamento de Carrera y Escalafón en Ecuador, para ser docentes se debe tener al menos un título de maestría o su equivalente. Al desagregar los resultados por sexo, las 
mujeres muestran un mayor porcentaje de preparación en cuanto a ingeniería/licenciatura y el $25 \%$ de mujeres alcanza el título de doctorado, mientras que solo el 6,66\% de los hombres posee uno.

En cuanto al estado civil de los docentes de la EPN, la mayoría son casados con el $57,38 \%$, seguido por el grupo de personas solteras que alcanzan el 34,43\%. Al desagregar los resultados por sexo los resultados se mantienen, pues el 56,3\% de las mujeres y el $57,8 \%$ de los hombres son casados.

Con relación a los hijos, el 55,7 \% tiene hijos y el 44,3 \% no los tiene; desagregado por sexo el 56,3\% de mujeres tiene hijos, mientras el 88,6\% de los hombres tiene hijos.

Con relación al tiempo de permanencia en la organización, el 66,67 \% de los docentes encuestados se encuentran trabajando en la institución en un rango de entre un mes hasta tres años, seguidos por el 21,3\% que tiene más de quince años laborando. Al desagregar los resultados por sexo, el 32,5 \% de las mujeres tienen un tiempo de permanencia de entre 0 a 3 años; no se registraron datos para las categorías de 3 a 5 años ni para el rango de 5 a 10 años, y para la categoría de 15 años en adelante el 15,4 \% corresponde a mujeres y el 84,6 \% a hombres, es decir, se evidencia que un mayor número de docentes mujeres se han integrado en los últimos tres años, lo que se corresponde con el hecho de que hay más docentes mujeres jóvenes que han decidido perseguir una carrera académica y han adquirido las competencias necesarias para hacerlo. Es evidente el progreso en la inclusión femenina, si se lo compara con el rango de 15 años en adelante. Dentro de esta pregunta cabe mencionar la situación laboral de los docentes en Ecuador; en los últimos años hubo una salida masiva de docentes universitarios (jubilación forzosa o voluntaria) por el proceso de jubilación de servidores públicos que se realizó bajo el amparo de los artículos 81 y 129 de la Ley Orgánica de Servicio Público (LOSEP), los artículos 288, 289, 290 del Reglamento de la Ley del Servicio Público, y la Disposición general novena de la Ley Orgánica de Educación Intercultural (LOEI) que crearon cuatro procesos de jubilación: jubilación obligatoria a partir de los 70 años, jubilación por enfermedad catastrófica, jubilación por invalidez y jubilación voluntaria con bonos del Estado.

En cuanto al tipo de relación laboral de los docentes en la EPN predomina el grupo de personas con nombramiento con el $52,46 \%$, y las personas que no tienen nombramiento 
representan el 45,9\%, lo que implica muchas veces tener un contrato de libre remoción, que afecta directamente a la estabilidad laboral del docente. Al desagregar los resultados por sexo se puede evidenciar que hay un mayor porcentaje de hombres con nombramiento $78,1 \%$, mientras que las mujeres solo alcanzan a un $21,9 \%$ lo que indica que el segundo grupo no cuenta con estabilidad laboral en su gran mayoría ni acceso a beneficios sociales, económicos y de promoción laboral que vienen vinculados al nombramiento.

Con respecto al cargo que ocupan, el 67,21\% se desempeña como docente investigador, el $23 \%$ como técnico docente y el 9,8 \% como coordinador de programa. Es importante mencionar que ningún Director de área o Decano llenó la encuesta; sin embargo, la información oficial que consta en la página web de la EPN, que según las leyes de transparencia del Ecuador debe informar a todos los grupos de interés sobre los datos de las dignidades y autoridades, se puede constatar que ningún puesto de director o decano está asignado a una mujer. Al desagregar los resultados por género, los hombres tienen un mayor porcentaje en el cargo de coordinador de programa -solo 1,6 \% de las mujeres tiene un puesto de coordinación-, mientras que como docentes y técnicos docentes las mujeres están más representadas; una vez más se constata que las mujeres no tienen acceso a puestos de poder, a pesar de existir una representación igualitaria entre los docentes contratados. Al analizar la pregunta de cargo como parte del total, se puede evidenciar que la representación de las mujeres es menor en todos los puestos desde el de coordinador de programa donde la diferencia porcentual es del $7 \%$ a favor de los hombres, por lo que se hace visible una barrera para las mujeres -techo de cristal-, pues aunque han logrado integrarse a la universidad como docentes o técnicas docentes no han sido vinculadas en puestos de gestión académica.

Matus-López (2015), en su artículo relacionado al techo de cristal, corrobora este hallazgo; primero que el discutir temas como acceso a puesto de poder/dirección es un tema sensible, que hay una diferencia de percepciones dependiendo de la edad y el sexo (los docentes hombres y mayores están de acuerdo con la asignación de puestos y responsabilidades) y las mujeres perciben la existencia de un techo de cristal y barreras a ascensos, es decir, las estadísticas de la presente investigación corroboran los descrito en varias investigaciones sobre la existencia de este problema y revela que no existe una mejoría significativa, a pesar de que en Ecuador existen leyes de inclusión, equidad e inclusive, acción afirmativa. 
Con relación a los salarios, la mayoría de los docentes gana entre $\$ 1000$ y $\$ 3000$ con el $68,88 \%$. Al desagregar por sexo, a partir de los $\$ 2000$ y hasta los $\$ 4000$, existen un mayor porcentaje mientras que en montos mayores se incrementa el número de hombres, lo cual puede ser debido a que los hombres ocupan puestos de mayor carga laboral e incluso no hay mujeres que ganen más de $\$ 4000$, pero sí un $15 \%$ de hombres que percibe este salario. Al considerar el sueldo en porcentajes, las mujeres son menores en todas las categorías de salario. Accenture (2017) realizó una investigación con más de 28.000 personas, distribuidas en tres generaciones, con una muestra en 29 países y los resultados revelan que persiste la brecha salarial entre hombres y mujeres, por cada $\$ 140$ dólares que gana un hombre, una mujer gana $\$ 100$.

\subsection{Preguntas de percepción}

En esta parte se presentan los resultados de las preguntas de percepción sobre clima laboral en las cuatro dimensiones seleccionadas: a) toma de decisiones, b) claridad organizacional, c) liderazgo y d) motivación con los resultados obtenidos para las preguntas planteadas, según la tabla 3.

\section{a). Toma de decisiones}

\section{En general, ¿considera que se toman en cuenta sus opiniones y comentarios?}

Esta pregunta tiene un promedio de 3,07 que se encuentra sobre el promedio aceptable e indica que la mayoría de veces los docentes consideran que se toman en cuenta sus opiniones y comentarios en el trabajo. Al desagregar los resultados por sexo no hay mujeres que consideren que siempre se toman en cuenta sus opiniones, a diferencia de un $8 \%$ de hombres que sí lo considera; además, un 31,25\% de mujeres considera que nunca o pocas veces se las toma en cuenta.

Dentro de los comentarios de la pregunta se señala que siempre se proporcionan comentarios para la mejora de la universidad, pero que estos no son tomados en cuenta por las autoridades; por otra parte, existe falta de comunicación y sociabilización en todos los aspectos de la universidad y los docentes opinan que se debe actuar sobre las políticas de educación para mejorar el sistema de retroalimentación de desempeño de los docentes por parte de los alumnos, ya que considera que la los resultados de evaluación a los docentes es sesgada y depende directamente de las calificación que los alumnos obtienen en la materia. 
2. ¿Considera que se le comunica asuntos importantes (por ejemplo, asuntos que modifican la vida laboral)?

Esta pregunta obtuvo un promedio de 3,07, lo que quiere decir que los docentes sienten que son de alguna manera consultados y comunicados sobre los cambios en la universidad. El 71,11 \% de hombres y el 43,75 \% de mujeres señalan que esto sucede siempre o casi siempre, de todas maneras, esta situación se muestra más favorable para los hombres.

En los comentarios de la pregunta los docentes mencionan que sí se les comunica sobre varios asuntos, pero no se lo hace a tiempo, por ejemplo, en el tema de renovación de contratos, capacitaciones y permisos.

\section{b). Claridad organizacional}

3. ¿Considera que se le proporciona la información necesaria para desempeñar sus labores, así como una retroalimentación adecuada?

Esta pregunta tiene un promedio de 3,15, lo que indica que los docentes, en la mayoría de casos, obtienen la información necesaria para desempeñar sus labores, son evaluados y reciben una retroalimentación del trabajo desempeñado. Al desagregar los resultados por sexo solo el 13,33 \% de las mujeres consideran que siempre tienen la información necesaria para desempeñar sus labores.

En cuanto a los comentarios, se menciona que la información para desempeñar el trabajo sí es recibida, pero muchas veces la retroalimentación es deficiente, ya que no hay seguimiento de las actividades; por otra parte, como una sugerencia se menciona que la mejor capacitación que podrían recibir los docentes para realizar su trabajo sería en pedagogía.

\section{4. ¿Considera que las responsabilidades de cada puesto están claramente definidas?}

Esta pregunta tiene un promedio de 3,15 lo que indica que, generalmente, los docentes consideran que las responsabilidades de su cargo están claramente determinadas. Al desagregar los resultados por sexo el 66,67 \% de hombres y el 81,25\% de mujeres considera que esto sucede así siempre o casi siempre.

Dentro de los comentarios se señala que siempre existe espacio para mejorar, pues todavía se puede precisar varios aspectos en cuanto a la definición de responsabilidades. 
5. ¿Considera que existe igualdad en la división de cargas de trabajo y asignación de responsabilidades?

Esta pregunta tiene un promedio de 2,82, e indica que los docentes consideran inequitativa la carga de trabajo y la asignación de responsabilidades. Esta pregunta se relaciona con la anterior que tiene un promedio superior, por lo que hay que aclarar la diferencia entre ambas. La pregunta anterior hace referencia a si los docentes tienen claras las funciones dentro de su cargo, mientras que esta pregunta se refiere a la igualdad en la asignación de dichas funciones. El 35,56 \% de los hombres y el 31,25 \% de las mujeres pocas veces consideran equitativa su carga laboral. Dentro de los comentarios se señala que no existe equidad en las responsabilidades incluso para personas que ocupan el mismo cargo, es decir, las responsabilidades de cada puesto están claramente definidas, pero existe una inequidad en la división de carga.

6. ¿Considera que los ascensos son otorgados a las personas que más se lo merecen y están más capacitados?

Esta pregunta tiene un promedio de 3,13, lo que indica que, en general, los docentes en la organización se encuentran conformes con los ascensos o puestos otorgados en la institución.

Respecto a los comentarios, se menciona que se priorizan los títulos de maestría y doctorado para otorgar puestos; sin embargo, se deja de lado otros aspectos importantes, como el trabajo de campo y la investigación. La tendencia en opiniones se mantiene en los resultados desagregados por género.

\section{c). Liderazgo}

7. ¿Los superiores o jefes le comunican sus expectativas?

Esta pregunta tiene un promedio de 3,08 e indica que en la mayoría de situaciones los docentes consideran que sus superiores les comunican las expectativas y resultados esperados sobre el trabajo y las tareas asignadas. Al desagregar los resultados por sexo, el 66,67\% de los hombres y el $62 \%$ de las mujeres consideran que siempre o casi siempre hay una comunicación adecuada, pero como en casi todas las preguntas, el porcentaje de mujeres que considera que pocas veces los jefes les comunican sus expectativas es mayor. 
Respecto a los comentarios, se señala que, a pesar de que los superiores sí comunican las expectativas, lo más importante son los resultados, sin tomar en cuenta el trabajo realizado para obtenerlos.

8. ¿Considera que los superiores son accesibles y es fácil hablar con ellos?

Esta pregunta tiene un promedio de 3,48, lo que indica que generalmente los docentes consideran que sus superiores son accesibles y pueden dirigirse a ellos cuando lo requieren. Más del $60 \%$ de los hombres y el $50 \%$ de las mujeres considera que casi siempre es de esta.

Respecto a los comentarios de la pregunta, se señala que muchas veces sí existe un trato prejuicioso e intolerante por parte de los superiores y una falta de proactividad, a pesar de ello, hay que destacar aquellos jefes que son comprensibles, con los cuales es admisible hablar y tienen un trato amable.

\section{d). Motivación}

9. ¿Considera que la organización se interesa por su bienestar personal y emocional no solo como empleado?

Esta pregunta tiene un promedio de 2,56, lo que indica que pocas veces los docentes consideran que la organización se interesa por su bienestar integral personal. Al desagregar los resultados por sexo, el 53,34 \% de los hombres y el 31,25\% de las mujeres considera que siempre o casi siempre perciben interés por parte de la organización; aun así, el porcentaje de personas que consideran que pocas veces o nunca sucede esto es alto, sobre todo para las mujeres que supera el $60 \%$.

Dentro de los comentarios de la pregunta se menciona que, a pesar de las quejas presentadas por malos tratos y comportamientos abusivos, estas no han sido resueltas y tampoco existen acciones claras por parte de la institución que demuestren un mayor interés por sus trabajadores.

\section{0. ¿Considera que debería ocupar un puesto superior dentro de la organización} por las responsabilidades que tiene?

Esta pregunta tiene un promedio de 2,97 e indica que, en general, los docentes no consideran que deberían ocupar un puesto superior al que actualmente tienen en la 
organización. El 42,32 \% de los hombres considera que algunas veces deberían ocupar un cargo superior, al igual que el 31,25 \% de las mujeres. El porcentaje de mujeres es menor, lo cual contrasta con lo establecido en el marco teórico de la investigación, donde se afirma que en muchas ocasiones la mujer no aspira a un cargo superior, puesto que le dificulta otras actividades, principalmente las tareas del hogar y su labor de madre; por otra parte, los hombres tienden a ser más competitivos y exigentes culturalmente, y aspiran a tener más tanto en el puesto que ocupan como en el salario que perciben.

Dentro de los comentarios de la pregunta se señala que, a pesar de no querer ocupar un puesto superior en la organización, sí se debería tratar con más respeto al personal en general. Otra de las razones por la cual los docentes creen que no deben ocupar un puesto superior es porque necesitan ganar experiencia y aún requieren un título de mayor nivel.

\section{1. ¿Se le otorgan reconocimientos por un trabajo bien desempeñado?}

Esta pregunta tiene un promedio de 2,39, que se encuentra por debajo del aceptable e indica que, generalmente, los docentes no reciben incentivos por un trabajo sobresaliente o reconocimientos por un trabajo mejor desempeñado. Más del $50 \%$, tanto de mujeres como de hombres, señalan que pocas veces o nunca reciben reconocimientos.

En cuanto a los comentarios de la pregunta se señala que, aparte de que no existen incentivos por un buen trabajo, muchas veces reciben amenazas por resultados que no se alcanzan y también que hay actividades por las que se debería recibir un reconocimiento (puede ser no económico), como publicaciones en revistas indexadas.

Para complementar este hallazgo, De la Rica (2007), en su investigación de segregación ocupacional y diferencias salariales por género para el período 1995-2002, con una muestra de 125.865 trabajadores en España, llega a una conclusión similar "una primera conclusión importante que podemos destacar es que, entre hombres y mujeres que trabajan en la misma ocupación y empresa y que tienen las mismas características observables en capital humano (medidas por edad y educación), los complementos salariales que reciben los hombres son entre un 27 y un $31 \%$ superiores a los que reciben las mujeres. Esta diferencia, es claramente el núcleo de las diferencias salariales ajustadas entre hombres y mujeres" (p. 12). 


\section{Conclusiones}

A nivel global, todas las preguntas de percepción recibieron una calificación satisfactoria. Sin embargo, al desagregar la información por género se puede observar que la mayoría de mujeres no están conformes o satisfechas con relación al clima organizacional y las cuatro dimensiones analizadas: toma de decisiones, claridad organizacional, liderazgo y motivación.

La percepción del ambiente laboral de los docentes en cuanto a si se toma en cuenta sus opiniones y se le proporciona información tanto para desempeñar sus labores como de asuntos importantes en el trabajo es favorable.

Con relación a la toma de decisiones, las mujeres perciben que sus opiniones casi no son tomadas en cuenta y no existe una comunicación adecuada sobre asuntos importantes. Con relación a la claridad organizacional, las mujeres perciben que no cuentan con la información necesaria para desempeñar sus labores y existe una sobrecarga de responsabilidades con relación a sus compañeros hombres. La dimensión de liderazgo es la única que es percibida de igual manera por hombres y mujeres, es decir, todos perciben que las expectativas son comunicadas adecuadamente y que los superiores son accesibles y es fácil hablar con ellos. Finalmente, con relación a la dimensión de motivación, la mayoría de mujeres percibe que la organización no se preocupa por su bienestar integral y la mayoría de ellas considera que no deberían ocupar un cargo más alto, situación que merece particular atención, toda vez que las mujeres son las que proporcionalmente tienen un mayor título (doctorado) y una mayor carga de trabajo; sin embargo, los hombres sí consideran que deberían ocupar un cargo más alto en la organización.

Además, después de analizar los estadísticos descriptivos, se evidencian problemas de discriminación laboral por género como acceso a posiciones de poder, mayor carga laboral y menor remuneración.

En lo referente a la organización, el 81,3\% de las mujeres y el $60 \%$ de los hombres llevan hasta tres años laborando en la institución, bajo una relación laboral con nombramiento (53 \%), donde existen un mayor porcentaje de hombres que de mujeres con nombramiento. Respecto al cargo, la mayoría se desempeña como docente investigador 
o técnico docente (78 \%); el cargo de coordinador de programa solo lo ejercen hombres, mientras que las mujeres predominan como docentes y técnicos docentes, lo que evidencia la discriminación laboral por género en la categoría de estabilidad laboral. Ningún director de área o decano es mujer, lo que evidencia la falta de acceso a puestos de poder y un evidente techo de cristal. En cuanto a salario, el 68,88 \% gana entre $\$ 1000$ y $\$ 3000$, pero al desagregar por sexo los resultados existen más mujeres que ganan salarios superiores a $\$ 2000$; sin embargo, a partir de los $\$ 4000$ se incrementa el número de hombres, lo cual se debe a que los hombres ocupan puestos de mayor carga laboral e incluso no hay mujeres que ganen más de $\$ 4000$, lo que indica una discriminación por brecha salarial. Es evidente que, a más de existir un clima organizacional inadecuado para las mujeres, existen prácticas de discriminación laboral por género.

\section{Referencias}

Accenture. (2017). Avanzando hacia la igualdad. Recuperado de https://www.accenture. com/es-es/careers/gender-equality-research-2016

Acker, S. (1995). Género y educación: reflexiones sociológicas sobre mujeres, enseñanza y feminismo. Madrid: Narcea Ediciones.

Arciniega, L. (julio-agosto, 2002). Compromiso organizacional México ¿Cómo hacer que la gente se ponga la camiseta? Dirección Estratégica, 11, 21-23.

Caligiore Corrales, I., \& Díaz Sosa, J. A. (2003). Clima organizacional y desempeño de los docentes en la ULA: Estudio de un caso. Revista Venezolana de Gerencia, 8(24), 644-658

Cardona-Echeverri, D. R., \& Cruz-Zambrano, R. (2014). Revisión de instrumentos de evaluación de clima organizacional. Estudios Gerenciales, 30(131), 184-189.

Castillo, L., Lengua, C., \& Pérez, P.A. (2011). Caracterización psicométrica de un instrumento de clima organizacional en el sector educativo universitario colombiano. International Journal of Psychological Research, 4(1), 40-47.

Chiang Vega, M. M., Núñez Partido, A., \& Huerta River, P. C. (2012). Relación del clima organizacional y la satisfacción laboral con los resultados, en grupos de docentes de instituciones de educación superior. Revista Icade, 72, 49-74.

Consejo Nacional para la Igualdad de Genero. (2014). Las mujeres en el Ecuador. En Análisis de los resultados de la Encuesta Nacional sobre Relaciones Familiares y Violencia de Género contra las Mujeres. Recuperado de http://www.unicef.org/ecuador/Violencia_de_Gnero.pdf 
De la Rica, S. (2007). Segregación ocupacional y diferencias salariales por género en España: 1995-2002. Madrid: Fundación de Estudios de Economía Aplicada, Serie Educación y Crecimiento: Cátedra FEDEA-Banco de España.

Fainstain, L., \& Perrotta, V. (2011). Guía para el Diágnóstico Organizacional con Perspectiva de Género. Recuperado de http://www.inmujeres.gub.uy/innovaportal/file/18680/1/ guia_disenodiagnostico_digital_1.pdf

Gacitúa, E., Sojo, C., \& Davis, S. (2000). Exclusión social y reducción de la pobreza en América Latina y el Caribe. San José: FLACSO, Banco Mundial.

García Solarte, M. (2011). Clima organizacional y su diagnóstico: una aproximación conceptual. Cuadernos de Administración, 25(42), 43-61.

Gil Lacruz, M. (2008). Género y educación, comentario al ensayo de Acker. Proyecto social: Revista de relaciones laborales, 12, 103-119.

Gonzáles, M. (2002). Feminismo, academia y cambio social. Revista Educación, 26(2), 169-182.

Guerra, C., Vargas, J., Castro, L., Plaza, H., \& Barrera, P. (2012). Percepción del clima escolar en estudiantes de enseñanza media de Valparaíso de colegios municipales, subvencionados y particulares. Estudios Pedagógicos, 38, 103-115.

Gül, H. (2008). Organizational Climate and Academic Staff's Perception on Climate Factors. Humanity \& Social Sciences Journal, 3(1), 37-48.

Halpin, A. W., \& Croft, D. B. (1962). The Organizational Climate of Schools. Chicago: Midwest Administration Center, University of Chicago.

Instituto Nacional de Mujeres. (2012). Modelo de equidad de género. Recuperado de https:// www.gob.mx/cms/uploads/attachment/file/27227/Guia-meg-2012.pdf

Instituto Nacional de Mujeres. (2013). Modelo de Calidad con Equidad de Género. Recuperado de http://www.inmujeres.gub.uy/innovaportal/file/25549/1/modelo_13febrero_.pdf

Jimenez, A. A. (2017) Diágnóstico De Prácticas De Discriminación Laboral Por Género En Las Universidades Públicas De Categoría A Del Distrito Metropolitano De Quito (tesis de pregrado para recibir el título de ingeniera empresarial). Escuela Politécnica Nacional, Quito.

Kurczyn, P. (2004). Acoso sexual y discriminación por maternidad en el trabajo. México: Universidad Nacional Autónoma de México.

Lopera Palacio, C. M. (2004). Antinomias, dilemas y falsas premisas que condicionan la gestión universitaria. Revista Mexicana de Investigación Educativa, 9(22), 617-635.

Merleau-Ponty, M. (1975). Fenomenología de la percepción. Barcelona: Península. 
Matus-López, M. (2015). Techo de cristal en la Universidad. Si no lo veo no lo creo. Revista Complutense de Educación, 26(3), 611-626.

Morán, T. E. \& Volkwein, J. F. (1992). The Cultural Approach to the Formation of Organizational Climate. Human Relations, 45(1), 19-47.

Murillo, P. \& Becerra, S. (2009). Las percepciones del clima escolar por directivos, docentes y alumnado mediante el empleo de "redes semánticas naturales». Su importancia en la gestión de los centros educativos. Revista de Educación, 350, 375-399

Oficina Internacional del Trabajo (OIT). (2013). Declaración relativa a los Principios y Derechos Fundamentales en el Trabajo. Recuperado de http://www.ilo.org/wcmsp5/groups/public/---ed_norm/---declaration/documents/publication/wcms_decl_fs_115_es.pdf

Peiró, J.M. (1995). Psicología de la organización. Madrid: Toran.

Rodríguez Mantilla, J. M, \& Fernández Díaz, M. J. (2015). Diseño y validación de un instrumento de medida del clima en centros de educación secundaria. Educación XXI, 18(1), 71-98.

Salazar Estrada, J. G., Guerrero Pupo, J. C., Machado Rodríguez, Y. B., \& Cañedo Andalia, R. (2009). Clima y cultura organizacional: dos componentes esenciales en la productividad laboral. Acimed, 20(4), 67-75.

Pérez de Maldonado, I., Maldonado Pérez, M., \& Bustamante Uzcátegui, S. (2006). Clima organizacional y gerencia: inductores del cambio organizacional. Investigación y postgrado, 21(2), 231-248.

Torrecilla, O. D. (2005). Clima organizacional y su relación con la productividad laboral. Documento de Cátedra. Argentina: Universidad Nacional de Córdoba-Facultad deficiencias Políticas y sociales. Recuperado de http://www.gestiopolis.com/organizacion-talento/intruduccion-al-clima-organizacional.htm.

Urrutia, J., \& Modrego, A. (2001). El gobierno de las universidades públicas. Recuperado de http://www.uib.es/premsa/juliol01/dia-06/ 361518.htm

Vega, M. M. C., Partido, A. N., \& Rivera, P. C. H. (2015). Efecto del clima organizacional en la autoeficacia de los docentes de instituciones de educación superior. Horizontes empresariales, 4(1), 61-74.

Vega, M. C., Rodrigo, M. J. M., \& Partido, A. N. (2010). Relaciones entre el clima organizacional y la satisfacción laboral. España: Universidad Pontificia de Comillas. 